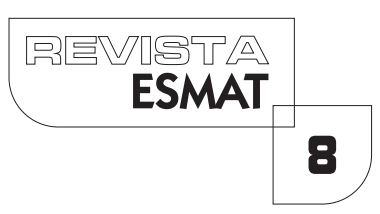

\title{
BIODIESEL E AGRICULTURA FAMILIAR NO TOCANTINS: UMA ANÁLISE A PARTIR DA TEORIA DOS CONJUNTOS FUZZY ${ }^{1}$
}

Vinícius Souza Ribeiro

Mestre em Desenvolvimento Regional (UFT, Brasil) e professor de economia agrícola do Instituto Federal de Educação Ciência e Tecnologia do Tocantins (IFTO).

Marcus Vinícius Alves Finco

PHD em Economia Agrícola pela Universitaet Hohenheim - Alemanha, professor adjunto da UFT.

\section{RESUMO}

A produção de biocombustíveis tem sido fortemente discutida no Brasil, levando o País a desenvolver políticas e implantar, em 2004, o Programa Nacional de Produção e Uso de Biodiesel (PNPB), com o objetivo de introduzir um biocombustível adequado às condições edafoclimáticas do País, e que tivesse como princípio a inclusão social da agricultura familiar e o desenvolvimento regional. Nesse contexto, o objetivo deste estudo é avaliar a eficácia do PNPB quanto à sua meta de inclusão dos agricultores familiares mais pobres na cadeia produtiva do biodiesel tocantinense, bem como estimar se a produção de soja para fins bioenergéticos gera aos agricultores familiares renda superior aos rendimentos provenientes dos outros plantios tradicionais do segmento no Brasil, assim como determinar e avaliar os impactos ambientais sobre a segurança alimentar, provenientes do cultivo da soja. Para a elaboração do presente estudo, foram utilizados dados primários, obtidos mediante a aplicação de questionários às 30 famílias produtoras de soja, e às 32 famílias não produtoras da oleaginosa, localizadas em 9 cidades do Centro do Tocantins; uma gama de indicadores socioeconômicos foi coletada entre os agricultores. A teoria dos conjuntos fuzzy, baseada na condição de vida dos agricultores, e um modelo não linear probit foram utilizados para avaliar a inclusão de famílias rurais pobres na cadeia do biodiesel. Os resultados preliminares mostram uma relação negativa entre o nível de privação das famílias e a adoção do cultivo de soja.

PALAVRAS-CHAVE: biodiesel, inclusão social, desenvolvimento regional

'O artigo é parte do Projeto "Agroenergia e Desenvolvimento Sustentável no Tocantins: uma Abordagem a partir de Sistemas Agrícolas e Rurais" (CNPq 40I598/20I I-0), liderado pelo segundo autor. 


\section{ABSTRACT}

Biofuel production has been greatly discussed in Brazil. In 2004, some debates lead the country to develop new policies and implement the National Biodiesel Use and Production Program (PNPB), with the aim of introducing a suitable biofuel for natural conditions of soil and climate of the country, which had social inclusion of family farming and regional development as a principle. In this context, the aim of this study is to evaluate the effectiveness of PNPB as to its goal of inclusion of the poorest farmers in the biodiesel production chain of Tocantins, as well as if the production of soybeans for bioenergy purposes generates higher income to family farmers compared to the traditional family plantings income in Brazil, and identify and assess the environmental impacts and impacts on food security from the cultivation of soybeans. Were used primary data through questionnaires, along with 30 soybean producing families and 32 non-producing families of oilseeds, located in 9 cities in the central Tocantins, a range of socio-economic indicators were collected. A fuzzy logic set theory based on living standard criteria and a non-linear probit model was applied to assess the inclusion of poor rural families in the biodiesel chain. Preliminary results point towards a negative relation between the family degree of deprivation and adoption of oil seed activity, for the soybean production.

KEYWORDS: biodiesel, social inclusion, regional development

\section{INTRODUÇÃO}

A preocupação quanto aos problemas ambientais, como a degradação e exaustão dos recursos naturais, bem como a poluição atmosférica e o aquecimento global, tem levado governos e cientistas a identificar alternativas e buscar soluções para mitigá-los. Os problemas ambientais ocorrem tanto nos países desenvolvidos como em países em desenvolvimento, tanto em áreas urbanas como em espaços rurais, e podem ser considerados como consequência dos atuais padrões de consumo e produção. É nesse contexto que, desde o início do século 21 , um debate internacional ganhou cada vez mais expressão e está presente na maior parte das conferências mundiais sobre desenvolvimento sustentável, a saber: biocombustíveis, seus prós e contras (FAO, 2008a; FAO, 2008c).

Em relação aos prós, os biocombustíveis podem auxiliar na mudança da matriz energética de um país, pelo uso de uma fonte mais limpa e renovável de energia. Com base nisso, os biocombustíveis também podem gerar externalidades positivas, como a manutenção da prestação de serviços 
ambientais, como o sequestro de carbono e a redução das emissões de carbono, por exemplo, (FAO, 2008d; FAO, 2008e). Do ponto de vista socioeconômico, os biocombustíveis podem alavancar o desenvolvimento regional e fomentar estratégias que reduzam e/ou aliviem a condição de pobreza, as chamadas estratégias "pró-pobres" (FAO, 2008b; FAO, 2008d).

Contudo, apesar das inúmeras vantagens, algumas questões surgiram em relação às possíveis externalidades negativas geradas pela produção de biocombustíveis. Estas incluem, por exemplo, a diminuição da produção e oferta de alimentos, bem como os impactos negativos sobre os serviços ambientais e as mudanças climáticas (FAO, 2008a). Quanto à questão da oferta de alimentos, a principal preocupação é a de que os biocombustíveis possam competir com as culturas alimentares. Essa competição por terra se torna um problema especialmente quando algumas das culturas que atualmente são cultivadas para a alimentação humana sejam redirecionadas para a produção de biocombustíveis (FAO, 2008b). Com a conversão de terras agrícolas para a produção de biocombustíveis, impactos negativos significativos sobre a segurança alimentar poderão ser observados, no que hoje é chamado de dilema "alimento versus combustível" ("food vs. fuel") (FAO, 2008a; FAO, 2008e).

Com base nessa discussão, e seguindo, portanto, o debate internacional sobre biocombustíveis, em 2004 o governo brasileiro lançou um programa de biodiesel chamado Programa Nacional de Produção e Uso de Biodiesel (PNPB). Tal programa tem como alguns de seus objetivos: implantar um programa sustentável, promovendo a chamada inclusão social ${ }^{2}$ dos agricultores familiares; garantir preços competitivos, qualidade e oferta de sementes oleaginosas, sem que isso interfira na produção de alimentos; produzir biodiesel a partir de óleos vegetais de diferentes espécies, concomitantemente à preservação do meio ambiente; e incrementar a renda agrícola dos agricultores familiares (PNPB, 2005).

Simultaneamente ao lançamento do PNBP, o governo federal, pelo Decreto no 5.297, de 6/I2/2004 (BRASIL, 2004), instituiu o Selo Combustível Social

\footnotetext{
${ }^{2} \mathrm{Na}$ dissertação, a "inclusão social" é entendida sob uma perspectiva multidimensionada, e não restrita somente à geração de emprego e renda (visão unidimensionada) como comumente é definida, superficialmente, em raríssimos programas de governo. Nesse sentido, é importante ressaltar que, a partir da abordagem conceitual adotada na dissertação, a inserção dos agricultores-familiares relativamente mais pobres no circuito produtivo do biodiesel, não é suficiente para que a inclusão social per si ocorra, mas sem dúvida oferece condições iniciais para que o fenômeno aconteça. Uma vez que o arco de sentidos da inclusão social vai muito além de mera inserção produtiva como preconiza o PNPB.
} 
(SCS) como instrumento de incentivo à inclusão social dos agricultores familiares na cadeia produtiva do biodiesel. Um dos critérios para a concessão do selo às empresas produtoras de biodiesel, por parte do Ministério de Desenvolvimento Agrário (MDA), é a compra de parte da matéria-prima proveniente dos estabelecimentos enquadrados no Programa de Nacional de Fortalecimento da Agricultura Familiar (PRONAF) com fins de produção do biocombustível.

As unidades industriais produtoras de biodiesel certificadas com o selo devem, segundo a Portaria $n^{\circ}$ 60, de 6 de setembro de 2012 (MDA, 20 I2), comprar um mínimo, em percentagem, de matéria-prima da agricultura familiar, percentual este que varia de acordo com a região no Brasil. Assim como, para fins de uso, manutenção e concessão do SCS devem celebrar previamente contratos com os agricultores familiares ou suas cooperativas agropecuárias, seguindo condições e critérios mínimos estabelecidos na Instrução Normativa nol, de 19/2/2009 (MDA, 2009), que garantam aos agricultores-familiares desde assistência técnica, de responsabilidade das empresas produtoras do biodiesel, até garantia de formação do preço, ex ante, da matéria-prima a ser entregue (com horizonte de variação positiva do preço vis-à-vis critérios préestabelecidos no contrato). Como contrapartida, as usinas têm facilidades de comercialização do biocombustível, incentivos tributários e melhores condições de financiamento via bancos públicos.

Essas estratégias lançadas pelo SCS inegavelmente têm caráter inovador, sobretudo se comparadas à falta de metas e estratégias de integração da agricultura familiar ao circuito produtivo dos biocombustíveis, como foi o caso do Proálcool. Porém, são necessários estudos mais aprofundados, a fim de se analisar até que ponto o PNPB, por meio do SCS, está de fato promovendo a inclusão social da agricultura familiar na cadeia produtiva, uma vez que, após aproximadamente uma década desde a sua implantação, os impactos e a eficácia do programa permanecem pouco claros.

Nesse sentido, dada a relevância do tema sob diferentes óticas (social, econômica, ambiental e alimentar), a dissertação, em questão, busca, primordialmente, analisar e compreender as relações entre a produção de sementes oleaginosas e a inclusão social, seguindo a concepção da Economia Verde Inclusiva ${ }^{4}$, tendo como objetivo geral a avaliação da eficácia do PNPB

${ }^{3}$ Tema amplamente discutido durante a Conferência Rio +20 , que ocorreu no mês de junho de 2012 na cidade do Rio de Janeiro, Brasil. 
quanto à inclusão dos agricultores-familiares mais pobres na cadeia produtiva do biodiesel tocantinense. Além de, secundariamente, estimar se a cultura da soja, com fins bioenergéticos, gera renda superior aos rendimentos provenientes dos plantios tradicionais da agricultura familiar tocantinense e quais os impactos de tal produção sobre a segurança alimentar familiar e o meio ambiente.

\section{ASPECTOS METODOLÓGICOSEÁREADE ESTUDO}

A metodologia dos sistemas agrícolas e rurais, adotada por Finco (20 I 0), foi utilizada no estudo. Tal metodologia descreve o sistema agrícola familiar como sendo composto principalmente por três setores: (I) agrícola, per se; (2) não agrícola; e (3) doméstico. Esses setores, segundo o autor, estão ligados por meio de relações internas, levando-se em consideração as necessidades, objetivos, problemas e expectativas dos agricultores-familiares sobre o futuro. Somado a isso, tal metodologia busca avaliar o sistema agrícola e rural de forma holística e sistêmica, pelos chamados indicadores de condição/padrão de vida (living standard indicators). Esses indicadores permitem profunda compreensão do funcionamento de um sistema agrícola e rural, uma vez que abordam aspectos econômicos, sociais, ambientais, demográficos, culturais e de saúde, dentre outros.

As fontes de informação utilizadas para realizar a pesquisa e obter os resultados incluem dados primários e inquéritos com agricultores familiares. É na região de transição do Cerrado para a Floresta Amazônica, dentro do estado do Tocantins, que a pesquisa foi realizada. $\bigcirc$ Tocantins está localizado na região norte do Brasil, e faz parte da chamada Amazônia Legal, e está situado numa zona de transição, apresentando clima e da vegetação da Floresta amazônica (I5\% do território) e Cerrado (85\% do território). Essa área de transição, chamada zona ecotonal, é o lar de comunidades tradicionais (agricultoresfamiliares, indígenas, e quilombolas) e inclui rica biodiversidade, a qual é responsável por inúmeros serviços ambientais. Por essa razão, estudos científicos e pesquisas na área são extremamente importantes (IBGE, 20 I 4).

Com base nisso, e a fim de obter as informações adequadas e relevantes para a pesquisa, uma macro região de estudo foi selecionada na parte centro-sul do estado do Tocantins, como se pode verificar na figura I.

Todavia, a maior parte dos dados secundários foi coletada na literatura sobre o tema. Os dados primários foram coletados no período compreendido entre anos de 2012 e 2013 , a partir de uma amostra de 62 famílias de agricultores familiares. Os dados colhidos foram baseados em entrevistas com os agricultores sobre atividades agrícolas da safra 20 II-2012, não agrícolas e 
domésticas, realizadas por um questionário estruturado. $O$ inquérito familiar foi realizado com agricultores-familiares que cultivavam sementes oleaginosas, e com agricultores-familiares que não as cultivavam ${ }^{4}$. Isso se fez necessário porque um dos objetivos da pesquisa é compreender a relação entre adoção do cultivo de sementes oleaginosas e pobreza rural.

Figura I. Região de estudo

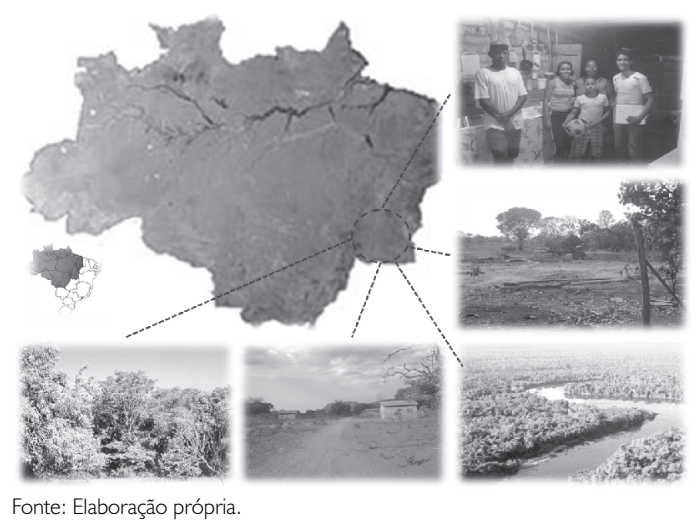

A lógica de conjuntos fuzzy e análises de regressão não linear probit também foram utilizadas no estudo. Como mencionado anteriormente, o objetivo da presente pesquisa foi analisar se houve, efetivamente, a inclusão das famílias de agricultores mais carentes no âmbito do PNPB. Para tal, foi construído um índice multidimensional de pobreza, denominado índice fuzzy de pobreza (IFP), que, além da renda familiar, levou em consideração outros indicadores de privação das famílias. Esse índice foi utilizado como proxy para determinar quais famílias eram consideradas as mais pobres na região em questão. A partir do conhecimento desse atributo, foi estimado um modelo probit com o objetivo de inferir se as famílias mais carentes estavam, efetivamente, participando da cadeia produtiva do biodiesel e, portanto, sendo socialmente incluídas na atividade, no estado do Tocantins.

A teoria dos conjuntos fuzzy foi escolhida porque permite a construção de um índice multidimensional de pobreza que (ao contrário de uma medida

\footnotetext{
${ }^{4} \mathrm{~A}$ listagem com os agricultores produtores de soja foi encaminhada pela usina de biodiesel. Para fins de determinação do grupo de não produtores de sementes oleaginosas, o procedimento adotado foi o de entrevistar também agricultores-familiares que apresentassem características semelhantes aos produtores de sementes oleaginosas e, preferencialmente, vizinhos de propriedade.
} 
tradicional de pobreza) não só leva em consideração a situação material dos agricultores-familiares, mas também capta as suas condições gerais de vida (MICELI, 1998) permitindo, com isso, que a relação entre inclusão social e produção de oleaginosas seja analisada e discutida com o devido aprofundamento. Muitas vezes, na abordagem tradicional, a pobreza é medida por uma linha de pobreza, ou seja, todos os indivíduos cuja renda não ultrapasse certo nível são declarados pobres (COSTA, 2002, DEUTSCH \& SILBER, 2005). Apesar de a abordagem tradicional apresentar seus méritos, como o de estabelecer os níveis de pobreza relativa e absoluta, ela não consegue capturar uma série de características que podem ser relevantes na compreensão de um fenômeno multidimensional como a pobreza.

Nesse contexto, a teoria dos conjuntos fuzzy aplicada a estudos de pobreza pode ser considerada uma abordagem adequada por permitir a construção de um índice que considera o grau de adesão do indivíduo, que pode variar entre 0 (sem adesão, ou não pobre) e I (total adesão ou completamente pobre). Além disso, fuzzy é uma análise ampla, incluindo uma série de indicadores de qualidade de vida, ao mesmo tempo em que adota ferramentas matemáticas que permitem captar nuanças de um fenômeno multidimensional (COSTA, 2002).

Todos esses aspectos metodológicos descritos conferem à pesquisa caráter de originalidade em relação às pesquisas desenvolvidas nacionalmente no segmento de socioeconomia rural, uma vez que utiliza procedimentos quantitativos e qualitativos, a partir de dados primários, para quantificar níveis de pobreza multidimensionados, analisando o fenômeno da (ex)inclusão social. Nesse aspecto, cabe destacar que o uso da teoria dos conjuntos fuzzy na área de ciências sociais (aplicadas) ${ }^{5}$, utilizada nesta dissertação, pode contribuir significativamente para que seja mais difundida e utilizada, sobretudo em pesquisas que abordem temáticas multidimensionadas ligadas à organização socioeconômica, tanto urbana quanto rural.

\section{RESULTADOS}

Com relação à geração de renda, os resultados apontaram a adoção do cultivo de oleaginosas para produção de biodiesel, no Tocantins gerou uma

\footnotetext{
${ }^{5}$ Até então, em âmbito nacional, fuzzy vem sendo majoritariamente utilizado em pesquisas realizadas pela Universidade de Campinas (UNICAMP), ligadas à área de ciência da computação e matemática, sobretudo quando se trabalha com a criação de inteligência artificial.
} 
renda superior aos rendimentos provenientes dos plantios tradicionais da agricultura familiar (no caso, arroz, feijão e milho). Porém, cabe a ressalva de que os atrasos observados no plantio da oleaginosa, resultado direto dos atrasos na liberação do financiamento por parte de um banco público, ocasionaram muitas perdas de produção refletindo diretamente na renda média gerada às famílias sojicultoras. Ocorrência esta abordada no presente estudo como eventual, que, em condições normais, não tenderia a prevalecer, como de fato observou-se para as safras posteriores (20 I 2 - I 3 e 20 I3- I 4).

Na ótica ambiental, apesar de a soja produzida estar causando os impactos negativos já conhecidos e esperados na Amazônia Legal, o modelo de conversão das áreas destinadas ao cultivo da soja preconizado pelos agricultores-familiares, sem desmatamento e com o uso de pastagens degradadas e áreas antes não utilizadas produtivamente, mitiga parte dos efeitos sobre a biodiversidade, mudanças climáticas, uso do solo e recursos hídricos. Quanto à segurança alimentar não houve elementos robustos o suficiente para afirmar que a produção de soja para fins bionergéticos tenha afetado negativamente a segurança alimentar das famílias, sob as dimensões de acesso e disponibilidade de alimentos, apontadas pela $\mathrm{FAO}(20 \mathrm{I})$.

Por meio dos 19 indicadores de pobreza construídos, a partir de dados primários, tabela I, foi calculado o IFP para cada uma das 62 famílias pesquisadas. A partir da figura 2 pôde-se observar que o grupo de famílias não produtor de soja apresentou, em média, valores superiores (mais próximos de I) em comparação ao grupo de famílias produtor de soja, apontando que, na média, o primeiro grupo contempla o grupo de famílias mais carentes, se comparado ao segundo grupo. Dessa forma, a priori, pôde-se auferir que o grupo de famílias de não produtores de soja é relativamente mais carente do que o grupo de famílias produtores da oleaginosa, sinalizando, ainda que de forma inicial, que a inclusão social é, no mínimo, duvidosa na região em questão.

\section{Figura 2. Indicadores de privação por grupos}

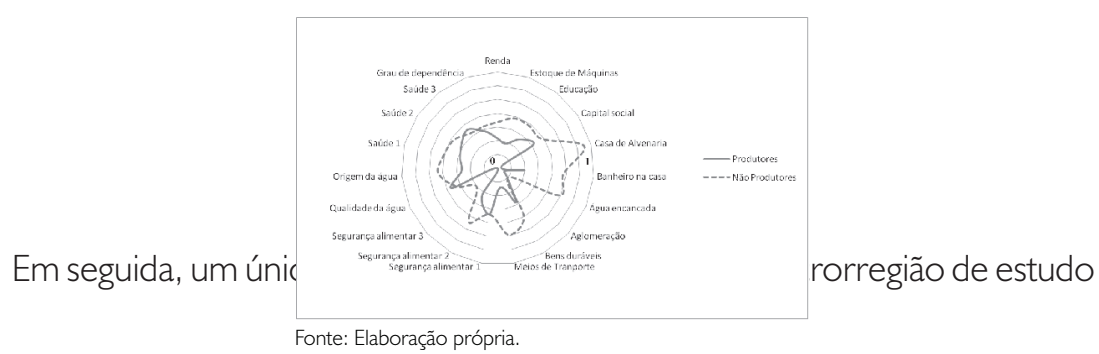


(tabela I) ${ }^{6}$, ou seja, compreendendo todos os agricultores-familiares (produtores e não produtores de soja). Após a estimativa do índice fuzzy de pobreza para a região, os agricultores-familiares foram separados em "pobres" e "não pobres", de acordo com seu próprio IFP. Com base nisso, os agricultoresfamiliares que possuíram, na média, um IFP acima 0,29 foram considerados pobres. Somado a isso, com o objetivo de compreender os vínculos entre o índice fuzzy de pobreza dos agricultores-familiares e a inclusão na cadeia produtiva do biodiesel, um modelo de regressão não linear probit foi formulado, a fim de estimar a relação entre a pobreza rural e a adoção do cultivo da soja.

\section{Tabela I. Índice de pobreza fuzzy}

\begin{tabular}{lcc}
\hline & \multicolumn{2}{c}{ Definição } \\
\cline { 2 - 3 } \multicolumn{1}{c}{ Indicadores ( ) } & $\begin{array}{c}\text { Média do Indicador Fuzzy } \\
\text { de pobreza }\end{array}$ & Pesos \\
\hline Renda familiar per capita & 0.633 & 0.022 \\
Estoque de máquinas & 0.613 & 0.023 \\
Anos de estudo & 0.524 & 0.031 \\
Capital social & 0.411 & 0.043 \\
Casa de alvenaria & 0.419 & 0.042 \\
Banheiro na Casa & 0.290 & 0.059 \\
Água encanada & 0.161 & 0.088 \\
Fator de aglomeração & 0.890 & 0.006 \\
Bens duráveis & 0.445 & 0.039 \\
Meios de transporte & 0.226 & 0.071 \\
Percebeu falta de alimentos no mercado & 0.468 & 0.036 \\
Deixou de fazer alguma das refeições & 0.290 & 0.059 \\
Arranjou-se com poucos alimentos & 0.032 & 0.165 \\
Qualidade da água & 0.294 & 0.059 \\
Origem da água & 0.403 & 0.044 \\
Autoavaliação das condições de saúde & 0.286 & 0.060 \\
Problema crônico de saúde & 0.444 & 0.039 \\
Avaliação do sistema de saúde usado & 0.484 & 0.035 \\
Grau de dependência & 0.189 & 0.080 \\
IFP (Índice Fuzzy de Pobreza) & 0.290 & \\
\hline
\end{tabular}

Fonte: Elaboração própria.

Como o objetivo do estudo foi avaliar a inclusão dos agricultores familiares

${ }^{6}$ Vale ressaltar que a teoria dos conjuntos fuzzy é aplicada por diferentes modelos (trapezoidal, linear e tradicional), bem como por estabelecimento de diferentes valores-âncora. É importante notar que quanto menor o número de agricultores privados com relação a determinado indicador de privação, maior é o peso deste indicador na composição do IFP. Isso acontece devido ao fato de que o sistema de pesos utilizados no presente estudo dá mais importância aos indicadores de privação associados com menores sintomas da pobreza, uma vez que os indivíduos têm, por natureza, forte sentimento de privação quando não possuem acesso a um bem ou serviço bastante difundido (MICELI, 1998). 
relativamente mais pobres na cadeia produtiva do biodiesel, ou seja, estimar a relação entre pobreza rural e adoção do cultivo de sementes oleaginosas, a especificação do modelo probit foi dada por:

$$
Y_{j}=a+\beta X_{j+} \mu_{j}(j=1, \ldots, n)
$$

Em que a variável dependente $Y$ refere-se à adoção de sementes oleaginosas ( $Y=1$ ) ou não adoção $(Y=0)$, $\alpha$ e $\beta$ são os parâmetros da equação, $\mu$ é o termo estocástico e a variável independente $X$ é uma variável dummy: I refere-se a quando uma família de agricultores familiares é considerada pobre; e 0 quando uma família não é considerada pobre (com base no índice fuzzy de pobreza, IFP, mencionado anteriormente) e, portanto:

$$
X=\left\{\begin{array}{l}
1 \text { se } X_{j}>0.290 \\
0 \text { se caso contrário }
\end{array}\right.
$$

A variável explicativa, expressada pelo índice fuzzy de pobreza, permite caracterizar a condição de vida dos agricultores-familiares na região em questão, valores mais baixos do IFP indicam uma condição de vida melhor. Assim, quando o sinal do coeficiente estimado a partir do modelo probit é positivo, a relação entre pobreza rural e inclusão na cadeia produtiva é direta, ou seja, a probabilidade de os agricultores familiares pobres adotarem o cultivo de sementes oleaginosas é maior do que os seus homólogos não pobres na mesma região. Portanto, a meta da inclusão social e a estratégia de desenvolvimento "pró-pobre" estão sendo eficazes. Mas quando o coeficiente for negativo, a relação é inversa, ou seja, os agricultores-familiares pobres têm menor probabilidade de adotar o cultivo de sementes oleaginosas do que os agricultores-familiares não pobres na mesma região e, portanto, a eficácia do PNPB, no que tange à meta da inclusão social, é, no mínimo, ambígua e duvidosa. Os resultados do modelo probit podem ser vistos na tabela 2 .

\section{Tabela 2. Resultados da regressão Probit}

$\begin{array}{lcccc} & \text { Coef. } & \text { Erro Padrão } & & \text { p } \\ \text { Itens } & .6985 & .2387 & 2.93 & 0.003 \\ \text { Constante } & -1.6432 & .3639 & -4.52 & 0.000 \\ \text { IFP } & & & 62 & \\ \text { Número de observações } & & & 22.7 & 0.000 \\ \text { LR } & & & & \\ \text { LR }\end{array}$

Pse ydode $R^{2}$-se observar que os coeficientes, na região do eśtúdo, são significativos Fonte: Elaboração própria. 
a 1\%. A probabilidade Qui-quadrado (×2) também é significativa a |\%. Percebese que o sinal do coeficiente IFP é negativo, ou seja, os agricultores-familiares pobres têm, em média, menor probabilidade de adotar o cultivo de sementes oleaginosas, quando comparados a seus pares que não são considerados pobres nessa mesma região. Aqui, é evidente que o desenvolvimento "própobre" não está ocorrendo, uma vez que os agricultores mais carentes não estão participando da cadeia produtiva do biodiesel e, portanto, a inclusão social defendida pelo PNPB não está sendo percebida.

Quando estimado o efeito marginal do coeficiente IFP, observa-se que ser um agricultor-familiar pobre nessa região em questão, por exemplo, diminui a probabilidade de adoção do cultivo de sementes oleaginosas (e, portanto, de não ser incluído na cadeia produtiva do biodiesel) em 58\%, em média, quando comparado a um agricultor-familiar não pobre.

Os resultados da pesquisa indicam, portanto, que o PNPB não está sendo eficaz, enquanto política de inclusão social, ou seja, as famílias de agricultores mais carentes não estão participando da cadeia produtiva do biodiesel. Sob outra ótica, o PNPB está atuando como uma política de exclusão social à medida que reduz significativamente a probabilidade de uma família carente fazer parte da cadeia produtiva do biocombustível em questão.

\section{CONCLUSÃO}

Apesar do fato de o PNPB possuir objetivos louváveis de promover a inclusão social e o desenvolvimento regional sustentável, os resultados apresentados demonstram ineficácia com relação à meta da inclusão social. Com base nisso, é imperativo dizer que o envolvimento de diferentes stakeholders, centros de pesquisa, empresas, governos locais e órgãos representativos da agricultura familiar parece ser uma condição sinequa non para superar as deficiências nos mecanismos estabelecidos pelo PNPB, com o objetivo de minimizar as lacunas da política nacional de biodiesel e, portanto, alcançar a inclusão social e o desenvolvimento regional sustentável.

Cabe ressaltar que este estudo é inédito na região, e os resultados são extremamente importantes para ajudar a obter um método apropriado para os governos regionais e nacionais para subsidiar a produção de energia limpa, sem agredir o meio ambiente local ou a produção de alimentos e, portanto, ajudar o Brasil a alcançar o desenvolvimento regional sustentável. Produção de sementes de óleo em pequena escala pode agora ser mais bem avaliada em outras partes da Amazônia Legal Brasileira, pois o estudo destaca um dos temas mais 
discutidos no debate sobre a bioenergia: produção de sementes oleaginosas e sua precedência sobre a inclusão socioprodutiva dos agricultores-familiares.

\section{REFERÊNCIAS}

BRASIL. Decreto Presidencial No 5.297 de 06 de Dezembro de 2004. $06 / 12 / 2004$ Disponível e m: <http://www.planalto.gov.br/ccivil_03/_ato20042006/2004/decreto/D5297.htm> (acessado em I 4 de agosto de 20 I 2).

COSTA, M. A Multidimensional Approach of the Measurement of Poverty. IRISS Working Paper Series n. 2002-05. 2002.

PNPB. 2005. Programa Nacional de Produção e Uso de Biodiesel. <http://www.biodiesel.gov.br/programa.html> (acessado em 12 de novembro de 20 I2).

FAO (Food and Agriculture Organization of the United Nations). Bioenergy and Food Security: The BEAFS Analytical Framework. Roma: FAO, 2010.

FAO (Food and Agriculture Organization of the United Nations). 2008b. Climate Change, Bioenergy and Food Security: Civil Society and Private Sector Perspectives. Disponível em: $<$ http://www.fao.org/fileadmin/user_upload/foodclimate/HLCdocs/HLC08inf-6-E.pdf> (acessado em 26 de outubro de 20 I 2).

FAO (Food and Agriculture Organization of the United Nations). 2008c. Climate Change, Bioenergy and Food Security: Options for Decision Makers identified by Expert Meetings. Disponível em: $<$ http://www.fao.org/fileadmin/user_upload/foodclimate/HLCdocs/HLC08inf-5-E.pdf > (acessado em 26 de outubro de 20 I 2).

FAO (Food and Agriculture Organization of the United Nations). 2008d. Bioenergy Policy, Markets and Trade and Food Security. Disponível em: < $\mathrm{ftp}: / /$ ftp.fao.org/docrep/fao/meeting/0 3/ai788e.pdf > (acessado em 26 de outubro de 2012).

FAO (Food and Agriculture Organization of the United Nations). 2008e. The 
State of Food and Agriculture. Biofuels: prospects, risks and opportunities. Disponível em:< http://www.fao.org/docrep/0II/i0l00e/i0l00e00.htm> (acessado em 26 de outubro de 20 I2).

DEUTSCH, J.; SILBER, J. Measuring Multidimensional Poverty: Na Empirical Comparison of Various Approaches. Review of Income and Wealth. Series 51 , n०51.2005.

MICELI, D. Measuring poverty using fuzzy sets. Discussionpaper $n^{\circ} 38$. National Centre for Social andEconomicModeling. Universityof Camberra. 1998. Disponível em: <http://natsem.edu.au/storage/dp38.pdf> (acessado em 20 de setembro de 20 I2).

MDA (Ministério do Desenvolvimento Agrário). Portaria ministerial n60 de 06 de Setembro de 2012.6/9/2012. . Instrução Normativa n I , de 19 de fevereiro de 2009. 19// 2/2009.

FINCO, M.V.A. Bioenergy Economics: An Analysis of Oil Seed Farming and Biodiesel Production in the Brazilian Savannah. Hohenheim, ALE, 20 I 0. 25 I p. Tese (Doutorado em Economia Rural) - Departamento de Economia Agrícola e Ciências Sociais nos Trópicos e Sub Trópicos, Universidade de Hohenheim, 2010 .

IBGE (Instituto brasileiro de Geografia e Estatística). IBGE Estados. 2014. Disponível em: <http://www.ibge.gov.br/estadosat/index.php> (acessado em 16 de fevereiro de 2014 ).

Recebido em: 23/04/2015

Aprovado em: 07/07/20 I 5 
